\title{
Impact of Awake Videothoracoscopic Surgery on Postoperative Lymphocyte Responses
}

\author{
Gianluca Vanni, MD, Federico Tacconi, MD, Francesco Sellitri, MD, \\ Vincenzo Ambrogi, MD, Tommaso Claudio Mineo, MD, and Eugenio Pompeo, MD
}

Department of Thoracic Surgery, Fondazione Policlinico Tor Vergata University, Rome, Italy

Background. Surgical stress and general anesthesia can have detrimental effects on postoperative immune function. We sought to comparatively evaluate postoperative lymphocytes response in patients undergoing videoassisted thoracoscopic surgery (VATS) under thoracic epidural or general anesthesia.

Methods. Between October 2008 and June 2009, 50 patients with nonmalignant pulmonary conditions were randomized to undergo VATS through either sole epidural anesthesia and spontaneous ventilation (awake group, $\mathbf{n}=$ 25) or general anesthesia with one-lung ventilation (control group, $n=25$ ). In both groups, assessment of total lymphocytes count and changes in proportion of lymphocyte subsets including CD19+, CD3+, CD4+, CD8+, CD4+:CD8+ ratio, and CD16+CD56+ (natural-killer cell) were evaluated by two-way analysis of variance test for repeated measures at baseline and postoperative days 1,2 , and 3 . The Mann-Whitney test was performed at each time point only for significant parameters at between-group analysis of variance.

$\mathrm{L}$ ymphocytes, and particularly natural-killer cells (CD16+/CD56+), are an important component of the immune system. This is mainly due to their cytotoxic activity against tumors and infections as well as to their ability to produce regulatory cytokines [1, 2]. It is widely documented that surgical stress, extensive tissue trauma, and general anesthesia may all reduce circulating lymphocytes and proportion of natural-killer cells [1-6]. Previous studies have suggested that an impairment of the immune function predisposes to postoperative infections [5-8]. Furthermore, reduced cytotoxic activity of peripheral-blood lymphocytes may increase the risk of tumor progression and metastases [4, 5, 9-13], and can even negatively affect postoperative healing processes.

Recent investigations in thoracic [14] and abdominal surgery [15-17] have shown that the immune function may be better preserved when surgical trauma is mini-

Accepted for publication April 19, 2010.

Presented at the Poster Session of the Forty-sixth Annual Meeting of The Society of Thoracic Surgeons, Fort Lauderdale, FL, Jan 25-27, 2010.

Address correspondence to Dr Mineo, Department of Thoracic Surgery, Fondazione Policlinico Tor Vergata University, Viale Oxford 81, 00133 Rome, Italy; e-mail: mineo@med.uniroma2.it.
Results. Comparisons of baseline data showed relatively homogeneous groups. Between-group analysis of variance was significant for proportion of natural-killer cells $(p=0.01)$. In particular, the control group disclosed a significantly lower median proportion of natural-killer cells as compared with the awake group on postoperative day 1 (5\% interquartile range [IQR]: $3 \%$ to $8 \%$ ] vs $12 \%$ [IQR: $8 \%$ to $14 \%$ ], $p=0.003)$ and $2(7 \%$ [IQR: $4 \%$ to $10 \%$ ] vs $11 \%$ [IQR: $8 \%$ to $21 \%$ ], $p=0.02$ ). Total lymphocyte count was significantly decreased in the control group only ( $p<0.00001)$. No difference was found between groups in the remaining lymphocyte subsets.

Conclusions. In this randomized study, awake VATS resulted in a lesser impact on postoperative lymphocyte responses than procedures performed under general anesthesia, as shown by the significant difference in postoperative proportion of natural-killer cells.

(Ann Thorac Surg 2010;90:973-8)

(C) 2010 by The Society of Thoracic Surgeons

mized. However, the protective role of video-assisted thoracoscopic surgery (VATS) in this setting is not yet elucidated and it is conceivable that whatever the surgical approach, every type of thoracic surgical procedure might have an impact on early postoperative immune response. Hypothetical reasons include surgical trauma, adverse effects of drugs employed during general anesthesia [18-20], and an impairment in natural-killer activity induced by one-lung ventilation (OLV) [8].

In 2001 we started an investigational clinical program entailing VATS carried out through sole thoracic epidural anesthesia in awake patients. Preliminary results with different types of surgical procedures have suggested that this novel surgical approach might reduce postoperative morbidity, hospital stay, and in-hospital costs [21-25].

We have now hypothesized that by avoiding adverse effects of general anesthesia, awake VATS could contribute to minimize at most the impact on early postoperative lymphocytes response. In this prospective, randomized study, we sought to comparatively evaluate postoperative lymphocyte response of simple VATS procedures performed through awake or general anesthesia. 


\section{Material and Methods}

\section{Study Design and Randomization Procedure}

The investigation was set up as a prospective randomized two-arm study (awake group versus control generalanesthesia group). The study was approved by the Ethical Committee of the Policlinico Tor Vergata Foundation. Mandatory prerequisite for enrollment was considered the diagnosis of a nonmalignant chest disease scheduled for VATS. Other inclusion criteria are detailed in Table 1.

The study was activated in October 2008 and was closed in June 2009 after the achievement of 50 patients. Prior to randomization, each patient was fully informed about the pros and cons of each type of surgical approach and gave her (his) written informed consent for enrollment in the study. Our aim was to assess the impact of general anesthesia or epidural anesthesia on postoperative lymphocyte response.

Primary outcome measure was the change in proportion of natural-killer lymphocytes at postoperative day 1 . This interval was chosen because in a previous pilot investigation we documented, at this time lag, the most significant change from baseline value. Trial size was determined by the number of patients necessary to yield, by a nonparametric two-sides test with a power of 0.8 and with an $\alpha$ error of 0.05 , a 1.5 -fold difference in median value of natural-killer percentage cells. In order to prevent influences due to the random allocation, the sequence was concealed from the surgeons and they were notified them at the operation time by telephone contact with the statistical department. Random assignment of patients to each group was carried out by computer (Excel software; Microsoft, Redmond, WA).

\section{Venous Blood Sampling Time Points}

Total lymphocytes count and changes in proportion of specific lymphocyte-subset including CD19+, CD3+, $\mathrm{CD} 4+, \mathrm{CD} 8+, \mathrm{CD} 4+: \mathrm{CD} 8+$ ratio, and natural-killer lymphocytes were measured at baseline just prior to the beginning of the operating session (7:00 a.m.), and at postoperative days 1, 2, and 3 at 7:00 a.m. Venous-blood samples were obtained through an antecubital vein and were transported to the Hematologic Department of our

\section{Table 1. Main Inclusion Criteria}

American Society of Anesthesiologists (ASA) score 1 (healthy subjects)

No use of steroids drugs from 2 months prior to operation to the postoperative period

No use of antiinflammatory drugs from 2 months prior to operation to the postoperative period

No use of $\beta$-adrenoceptor agonist drugs from 2 months prior to operation to the postoperative period

No clinical suspicion of active pleural-pulmonary infection

No previous history of cancer

No autoimmune diseases

No diabetes mellitus or endocrine disorders

Patient's acceptance of the assigned study group
Institution for immediate, real-time tests without need of storage.

Total lymphocytes were measured with a cell counter (Coulter Beckmann, MedLab, Cupertino, CA). Lymphocyte-subset including CD19+, CD3+, CD4+, CD8+, $\mathrm{CD} 4+: \mathrm{CD} 8+$ ratio, and natural-killer, were analyzed by FACSCalibur three-color flow cytometry (Immunocytometry System, BD Biosciences, Franklin Lakes, NJ) with antibodies specific to the cell markers. The blood samples were incubated for 30 minutes with monoclonal antibodies at $4^{\circ} \mathrm{C}$. The samples were processed with a coulter, which lyses the erythrocytes, and stabilizes and fixes the leukocytes. The percentage of lymphocytes was evaluated through differential gating after triple-color staining.

\section{Anesthesia Technique and Postoperative Management}

In both study groups, venous line (16 gauge) and radial artery catheter were placed. In awake patients, the thoracic epidural catheter was inserted at the $\mathrm{T} 4$ level by a loss-of-resistance technique, and a bolus of ropivacaine $(0.5 \%)$ plus sufentanyl $(5 \mu \mathrm{g})$ was immediately administered after placement. Subsequently, continuous infusion of ropivacaine $(0.2 \%)$ at a rate of $5 \mathrm{~mL} /$ hour was started 20 minutes prior to operation. In the meantime, the patient was placed in lateral decubitus with the hemithorax targeted for operation in a nondependent position. If necessary, additional injection of local anesthetics (2 to 5 $\mathrm{mL}$ of ropivacaine $[7.5 \%]$ plus lidocaine $[2 \%]$ ) was used to reinforce analgesia at the incision sites. During the operation, oxygen was delivered through a Venturi mask at the minimum regimen to keep arterial saturation above $90 \%$. All surgical procedures were conducted by the same surgeons, anesthesiologists, and nursing staff.

In the control group, general anesthesia was induced by continuous intravenous administration of propofol and sufentanyl $0.5(\mu \mathrm{g} / \mathrm{kg})$, while cis-atracurium $(0.15$ $\mathrm{mg} / \mathrm{kg}$ ) was used for muscle relaxation. Anesthesia was maintained by means of a target-control infusion of propofol, with a target-concentration at effector site of 4 $\mu \mathrm{g} / \mathrm{mL}$. No inhalational agent was used. For selective ventilation, a left-side double lumen tube was used in all instances. One-lung ventilation was started immediately prior to positioning the patient on lateral decubitus. Pressure-controlled tidal ventilation was set with a peak airway pressure not exceeding 25 to $30 \mathrm{~cm} \mathrm{H}_{2} \mathrm{O}$ and a positive end-expiratory pressure of $5 \mathrm{~cm} \mathrm{H}_{2} \mathrm{O}$. After the procedure, patients were transferred to the recovery room and weaned after an early extubation protocol.

During the procedure, fluid infusion was set at $1.5 \mathrm{~mL}$. $\mathrm{kg}^{-1} \cdot$ hour $^{-1}$ of normal saline and Ringer's solution in both groups. In the awake group, fluid infusion was continued until 2 hours after surgery and oral intake was immediately allowed. In the control group, fluid infusion was stopped 24 hours after the operation. No significant differences in fluid balance was found between the two groups as indicated by similar urinary output, as well as no difference in creatinine level in the perioperative period.

Postoperative analgesia was assured through continuous intravenous administration of tramadol (200 to 300 
$\mathrm{mg} / 24$ hours at a rate of $2 \mathrm{~mL} /$ hour) by an elastomeric device. No antiinflammatory drugs were given postoperatively in either study group.

\section{Surgical Techniques}

All patients were placed in full lateral decubitus position with slight trunk elevation and splitting of the intercostal spaces. The operative techniques in awake patients did not differ from the equivalent procedure performed under general anesthesia and OLV. Lung volume reduction surgery was performed by a nonresectional technique [16] through 4 flexible trocars and plication of the most emphysematous lung regions was carried out by means of "no knife" endostaplers. Bullectomies and wedge resections were made through a 3-trocars' access [14, 17]. Mediastinal and (or) pleural biopsies were accomplished through a single $20-\mathrm{mm}$ trocar by means of a dedicated spoon-shaped forceps. One $28 \mathrm{Ch}$ chest drainage was placed at the end of each procedure while complete lung reexpansion was always controlled under thoracoscopic vision. Antibiotic prophylaxis entailed intravenous cefuroxime $(2 \mathrm{~g})$ twice a day, until postoperative day 2 . Criteria for chest tube removal were standardized, and included no evidence of air leak after 3 hours of tube clamping, serous fluid loss less than $200 \mathrm{~mL} / 24$ hours, and absence of pneumothorax at a chest X-ray.

\section{Statistical Analysis}

Data are presented as median and interquartile range (IQR). Two-way analysis of variance test for repeated measures was employed to analyze results at baseline and postoperative days 1,2 and 3. Variables showing significant $(p \leq 0.05)$ between-group difference were subsequently analyzed by means of the Mann-Whitney $U$ test at each time point.

\section{Results}

\section{Baseline Data and Surgical Results}

Out of the 59 patients initially considered for enrollment, 3 patients refused consent because they preferred general anesthesia. After randomization, 6 patients ( 3 for each group) were excluded because of conversion to thoracotomy under general anesthesia $(\mathrm{n}=2)$, diagnosis of unexpected malignant nodules $(\mathrm{n}=2)$, postoperative air leakage longer than 7 days $(\mathrm{n}=1)$, and severe ventricular arrhythmia requiring electric conversion in the immediate postoperative period $(n=1)$. As a consequence the study group consisted of 50 patients, who were divided into the two study groups. A comprehensive summary of demographics, preoperative and perioperative data is depicted in Table 2, which shows that study groups were adequately matched in terms of demographics, baseline findings, and distribution of the surgical procedures.

There were 16 lung nodules that included pulmonary hamartomas $(n=13)$, granulomas $(n=2)$, and neuroendocrine cell hyperplasia $(n=1)$. Pleural biopsies revealed simple hyperplasia in 5 instances, benign fibrous tumor in 1 case, and lipoma in another. Antracotic lymph nodes were found in the 2 patients undergoing mediastinal sampling.

Median operative time was similar in both groups and neither intraoperative complication nor technical difficulty occurred in any patient. Minor complications included air leakage lasting more than 5 days ( 1 in the awake group and 2 in the control group), and a contralateral lung atelectasis requiring fiberoptic bronchoscopy (1 patient in the control group). Hospital stay was not different between study groups (Table 2).

Table 2. Main Baseline and Operative Findings in Study Groups. Data Are Expressed as Median and Interquartile Range (Within Brackets). Significance Was Tested With the Mann-Whitney U Test

\begin{tabular}{|c|c|c|c|}
\hline Variable & Awake Group $(\mathrm{n}=25)$ & Control Group $(n=25)$ & $p$ Value Intergroup \\
\hline \multicolumn{4}{|l|}{ Baseline findings: } \\
\hline Age (years) & $57(51-62)$ & $52(46.5-60.5)$ & 0.36 \\
\hline M:F (ratio) & $15: 10$ & $16: 9$ & 1 \\
\hline Right to left (ratio) & $15: 10$ & 14:11 & 1 \\
\hline \multicolumn{4}{|l|}{ Preoperative diagnosis } \\
\hline Pulmonary emphysema (n.pts) & 12 & 6 & 0.13 \\
\hline Pulmonary nodule (n.pts) & 6 & 10 & 0.36 \\
\hline Pleural thickening (n.pts) & 3 & 4 & 1 \\
\hline Pneumothorax (n.pts) & 2 & 3 & 1 \\
\hline Mediastinal lymphadenopathy (n.pts) & 1 & 1 & 1 \\
\hline Pulmonary fibrosis (n.pts) & 1 & 1 & 1 \\
\hline \multicolumn{4}{|l|}{ Perioperative-postoperative results: } \\
\hline Operative time (minutes) & $44(43-47)$ & $43.5(41-46)$ & 0.24 \\
\hline Minor complication (rate) & $1 / 25$ & $3 / 25$ & 0.6 \\
\hline Hospital stay (days) & $3.3(2-9)$ & $3.2(2-10)$ & 0.8 \\
\hline
\end{tabular}

n.pts $=$ number of patients. 


\section{Total Lymphocytes and Lymphocyte Subsets Changes}

Table 3 shows a comprehensive summary of the study results. At baseline, total lymphocytes count and proportion of specific lymphocyte subsets were similar in the two groups. Between-group analysis of variance was significant for proportion of natural-killer cells $(p=0.01)$, which decreased from baseline value in the control group only (within-group analysis, $p=0.02$ ). In particular, a significantly lower proportion of natural-killer cells was found in the control group on postoperative day $1(5 \%$ [IQR: $3 \%$ to $8 \%$ ] vs $12 \%$ [IQR: $8 \%$ to $14 \%$ ], $p=0.003$ ) and day 2 ( $7 \%$ [IQR: $4 \%$ to $10 \%$ ] vs $11 \%$ [IQR: $8 \%$ to $21 \%$ ], $p=$ $0.02)$. Yet, within-group analysis of variance showed a significant difference in total lymphocyte count in the control group only $(p<0.0001)$. No difference was found between groups in the remaining lymphocyte subsets.

\section{Comment}

Preserved lymphocyte function contributes to improve resistance to postoperative infections [4-6].

In addition, natural-killer cells represent a possible first line of defense against intravascular tumor spread because, in absence of major histocompatibility-complex restriction, different types of malignant cells can be killed by these cells in a nonspecific way [5, 9, 11-13]. Furthermore, an impairment in immune function and lymphocyte activity has shown to be associated with several adverse effects including an increased incidence of post- operative infections and an increased risk of tumor growth [4-6, 9-13].

In our study, awake VATS resulted in a reduced impact on postoperative lymphocyte response and particularly on the proportion of natural-killer cells, when compared with equivalent procedures performed under general anesthesia. These findings suggest a different pattern of lymphocyte response between the two groups. In particular, our data showed that total lymphocyte count and the proportion of natural killer cells decreased postoperatively in the control group while remained unchanged in patients receiving awake anesthesia. Although various factors could have contributed to influence this effect, the randomization and the relatively well-matched baseline study data led us to hypothesize that the type of anesthesia has probably played a major role in postoperative changes of lymphocyte response. Available data regarding the effect of epidural anesthesia on the immune function are still controversial. Papadima and colleagues [26] have found that epidural anesthesia did not suppress postoperative lymphocyte apoptosis in patients receiving major abdominal surgery. Beilin and colleagues [27] showed that epidural analgesia may protect against late ( $<72$ hours) postoperative lymphocyte suppression, but this effect was not found when restricting the analysis to the early postoperative period. Furthermore, Yokoyama and colleagues [6] found that epidural anesthesia is associated with a transient reduction in natural-killer activity that has been considered unrelated to the pain

Table 3. Preoperative and Postoperative Responses of Total Lymphocytes and Lymphocyte Subsets Evaluated by the Two-Way Analysis of Variance Test for Repeated Measures. Data are Expressed as Median and Interquartile Range (Within Brackets)

\begin{tabular}{|c|c|c|c|c|c|c|c|}
\hline & Baseline & Day 1 & Day 2 & Day 3 & $\begin{array}{l}\text { Within } \\
\text { Group } \\
p \text { Value }\end{array}$ & $\begin{array}{l}\text { Between } \\
\text { Group } \\
p \text { Value }\end{array}$ & $\begin{array}{c}\text { Interaction } \\
p \text { Value }\end{array}$ \\
\hline \multicolumn{8}{|c|}{ Total lymphocytes (n $10^{9} / \mathrm{L}$ ): } \\
\hline Awake group & $1.82(1.42-2.44)$ & $1.72(1.47-2.02)$ & $1.53(1.31-1.92)$ & $1.7(1.23-1.95)$ & 0.32 & 0.77 & 0.01 \\
\hline Control group & $2.03(1.78-2.7)$ & $1.56(1.13-1.87)$ & $1.52(1.25-1.93)$ & $1.25(1.04-1.62)$ & $<0.00001$ & & \\
\hline \multicolumn{8}{|l|}{ CD19+ $(\%):$} \\
\hline Awake group & $11(7-13.5)$ & $11(8-17)$ & $12(9-16)$ & $8(6-18)$ & 0.41 & 0.25 & 0.29 \\
\hline Control group & $11(7-15)$ & $12(8-14)$ & $9(7-14)$ & $9.5(7-15)$ & 0.79 & & \\
\hline \multicolumn{8}{|l|}{ CD3+ $(\%):$} \\
\hline Awake group & $69(61.5-75)$ & $67(62-64)$ & $60(55-73)$ & $73(61-76)$ & 0.92 & 0.62 & 0.79 \\
\hline Control group & $72(65-76)$ & $71(60-77)$ & $75(65-80)$ & $69.5(66-80)$ & 0.54 & & \\
\hline \multicolumn{8}{|l|}{$\mathrm{CD} 4+(\%):$} \\
\hline Awake group & $50(40.5-53.5)$ & $47(33-52)$ & $45(33.5-56)$ & $49(44-52)$ & 0.34 & 0.99 & 0.34 \\
\hline Control group & $46(40-55)$ & $44(39-52)$ & $49(38-53)$ & $47(42-52)$ & 0.44 & & \\
\hline \multicolumn{8}{|l|}{ CD8+ $(\%):$} \\
\hline Awake group & $20.5(15-26.5)$ & $20(16-31)$ & $18(13-25)$ & $20(15-25)$ & 0.70 & 0.51 & 0.73 \\
\hline Control group & $25(17-29)$ & $25(16-30)$ & $19(15-31)$ & $23(15-29)$ & 0.61 & & \\
\hline \multicolumn{8}{|c|}{ CD4+:CD8+ (ratio): } \\
\hline Awake group & $2.4(1.4-3.4)$ & $2.8(1.6-3.5)$ & $3.3(1.9-4.2)$ & $2.5(1.8-3.2)$ & 0.85 & 0.27 & 0.72 \\
\hline Control group & $1.7(1-3.1)$ & $1.7(1.1-2.6)$ & $2.1(1.6-3.4)$ & $1.95(1.8-3.2)$ & 0.80 & & \\
\hline \multicolumn{8}{|l|}{ Natural killer (\%): } \\
\hline Awake group & $12(8-14)$ & $12(8-14)$ & $11(8-21)$ & $12(8-13)$ & 0.20 & 0.01 & 0.11 \\
\hline Control group & $8(5-12)$ & $5(3-8)$ & $7(4-10)$ & $7(2-14)$ & 0.02 & & \\
\hline
\end{tabular}

CD3 = T-lymphocyte; 
level as well as to the underlying disease. The same authors hypothesized that natural-killer cells inhibition may be partially modulated both by sympathetic block and cortisol plasma increase. Infusion of epinephrine enhances natural-killer activity [28] and this effect can be inhibited by oral administration of a $\beta$-adrenoceptor antagonist [29]. These effects might be explained by the presence of adrenergic-specific receptors expressed by lymphocytes placed in close proximity of sympathetic nerve endings [30].

Drugs administered to patients during general anesthesia may also affect lymphocyte response [18]. In particular, opioids may depress immunity [18-20] although some effect can also be related to nonopioid anesthetics and propofol. Pirttikangas and colleagues [20] found a higher percentage of CD4+ cells after anesthesia with propofol in comparison with conventional combined anesthesia. Another poorly investigated field regards the potential interaction between cisatracurium and immunologic response.

Avoidance of OLV could also have contributed to the more physiologic lymphocyte response observed in awake patients. Indeed, in 1993, Tonnesen and colleagues [8] first reported that in patients undergoing major thoracic surgery under general anesthesia, OLV was associated with an immediate decrease in natural-killer activity. Recent studies [31-33], have shown that OLV can trigger a series of complex oxidative changes in both the ventilated and nonventilated lung, which eventually result in a compartmental release of proinflammatory mediators including interleukin 6 and 8, and tumor necrosis factor- $\alpha$. The local activation of proinflammatory mediators and the subsequent spillover into the systemic circulation could act as an important modulator of postoperative lymphocyte response. As the hypothalamicpituitary-axis activity is enhanced by some circulating cytokines including interleukin-6 [2], OLV may interfere with natural-killer activity by a transient increase of cortisol plasma level, which is one of the main modulators of lymphocyte apoptosis and function.

In our study, the proportion of natural-killer cells decreased postoperatively in the control group whereas it remained relatively stable in the awake group. This might reveal a potential advantage of awake thoracic surgery. Other potential advantages of this globally minimally invasive approach have been previously shown by our group [21-25] and include easy accomplishment of fast-track surgery programs, prompt resumption of common daily life activities, and reduced in-hospital costs.

We acknowledge some limitations of our study that include the small cohort and the nonhomogeneous types of surgical procedures. Nonetheless, the trial sample size was determined a priori to assure a statistically acceptable power in detecting intergroup differences in results; yet, study groups were well matched in demographics, and preoperative and surgical data. Another limitation might be considered the avoidance of epidural anesthesia in the control group. However, we have considered it nonethical to perform epidural catheterization and general anesthesia in patients undergoing simple VATS procedures. This choice is also justified from some reports in the literature [34-36]. Finally, our current analysis is still limited to quantitative assessment and relative proportion of circulating lymphocyte subsets, whereas no data on lymphocyte activity as well as circulating inflammation biomarkers and endocrine response are presented in this study.

In conclusion, awake VATS is a promising and potentially revolutionary novel surgical approach that warrants further clinical investigation. Our current findings might reveal practical relevance once corroborated by larger studies showing that the higher postoperative lymphocytes depletion observed in the general anesthesia group is associated with an increased risk of postoperative infections and (or) tumor progression.

\section{References}

1. Kutza J, Gratz I, Afshar M, Murasko DM. The effects of general anesthesia and surgery on basal and interferon stimulated Natural killer cell activity of humans. Anesth Analg 1997;85:918-23.

2. Desborough JP. The stress response to trauma and surgery. Br J Anaesth 2000;85:109-17.

3. Schilling T, Kozian Alf, Huth C, et al. The pulmonary immune effects of mechanical ventilation in patients undergoing thoracic surgery. Anesth Analg 2005;101:957-65.

4. Meakins JL. Surgeons, surgery, and immunomodulation. Arch Surg 1991;126:494-8.

5. Koltun WA, Bloomer MM, Tilberg AF, et al. Awake epidural anesthesia is associated with improved natural killer cell cytotoxicity and a reduced stress response. Am J Surg 1996;171:68-72.

6. Yokoyama M, Itano $\mathrm{Y}$, Katayama $\mathrm{H}$, et al. The effects of continuous epidural anesthesia and analgesia on stress response and immune function in patients undergoing radical esophagectomy. Anesth Analg 2005;101:1521-7.

7. Ahlers O, Nachtigall I, Lenze J, et al. Intraoperative thoracic epidural anaesthesia attenuates stress-induced immunosuppression in patients undergoing major abdominal surgery. Br J Anaesth 2008;101:781-7.

8. Tonnesen E, Hohndorf K, Lerbjerg G, Christensen NJ, Huttel $\mathrm{S}$, Andersen K. Immunological and hormonal responses to lung surgery during one-lung ventilation. Eur J Anaesth 1993;10:189-95.

9. Talmadge JE, Meyers KM, Prieur DJ, Starkey JR. Role of NK cells in tumour growth and metastasis in beige mice. Nature 1980;284:622-5.

10. Herberman RB, Ortaldo JR. Natural Killer cells: their role in defenses against disease. Science 1981;241:24-30.

11. Imai K, Matsuyama S, Miyake S, Suga K, Nakachi K. Natural cytotoxic activity of peripheral-blood lymphocytes and cancer incidence: an 11-year follow-up study of a general population. Lancet 2000;356(9244):1795-9.

12. Colacchio TA, Yeager MP, Hildebrandt LW. Perioperative immunomodulation in cancer surgery. Am J Surg 1994;167: 174-9.

13. Trinchieri G, Perussia B. Human natural killer cells: biologic and pathologic aspetcs. Lab Invest 1984;50:489-513.

14. Whitson BA, D'Cunha J, Andrade RS, et al. Thoracoscopic versus thoracotomy approaches to lobectomy: differential impairment of cellular immunity. Ann Thorac Surg 2008;86: 1735-44.

15. Lee SW, Gleason N, Blanco I, Asi ZK, Whelan RL. Higher colon cancer tumor proliferative index and lower tumor cell death rate in mice undergoing laparotomy versus insufflation. Surg Endosc 2002;16:36-9.

16. Allendorf JD, Bessler M, Horvath KD, Marvin MR, Laird DA, Whelan RL. Increased tumor establishment and growth after 
open vs laparoscopic surgery in mice may be related to differences in postoperative T-cell function. Surg Endosc 1999;13:233-5.

17. Lee SW, Feingold DL, Carter JJ, et al. Peritoneal macrophage and blood monocyte functions after open and laparoscopicassisted cecectomy in rats. Surg Endosc 2003;17:1996-2002.

18. Tubaro E, Borelli C, Croce C, Cavallo G, Santiangeli C. Effect of morphine on resistance to infection. J Infect Dis 1983;148: 656-66.

19. Yeager MP, Colacchio TA, Yu CT, et al. Morphine inhibits spontaneous and cytokine-enhanced natural killer cell function in human volunteers. Anesthesiology 1995;83:500-8.

20. Pirttikangas CO, Perttilä J, Salo M, Vainio O, Liukko-Sipi S. Propofol infusion anaesthesia and immune response in minor surgery. Anaesthesia 1994;49:13-6.

21. Pompeo E, Mineo TC. Awake operative videothoracoscopic pulmonary resections. Thorac Surg Clin 2008;18:311-20.

22. Pompeo E, Tacconi F, Mineo D, Mineo TC. The role of awake video-assisted thoracoscopic surgery in spontaneous pneumothorax. J Thorac Cardiovasc Surg 2007;133:786-90.

23. Mineo TC, Pompeo E, Mineo D, Tacconi F, Marino M, Sabato AF. Awake nonresectional lung volume reduction surgery. Ann Surg 2006;243:131-6.

24. Pompeo E, Mineo D, Rogliani P, Sabato AF, Mineo TC. Feasibility and results of awake thoracoscopic resection of solitary pulmonary nodules. Ann Thorac Surg 2004;78:1761-8.

25. Mineo TC. Epidural anesthesia in awake thoracic surgery. Eur J Cardiothorac Surg 2007;32:13-9.

26. Papadima A, Boutsikou M, Lagoudianakis EE, et al. Lymphocyte apoptosis after major abdominal surgery is not influenced by anesthetic technique: a comparative study of general anesthesia versus combined general and epidural analgesia. J Clin Anesth 2009;21:414-21.
27. Beilin B, Shavit $Y$, Trabekin E, et al. The effects of postoperative pain management on immune response to surgery. Anesth Analg 2003;97:822-7.

28. Kappel M, Tvede N, Galbo H, et al. Evidence that the effect of physical exercise on NK cell activity is mediated by epinephrine. J Appl Physiol 1991;70:2530-4.

29. Bachen EA, Manuck SB, Cohen S, et al. Adrenergic blockade ameliorates cellular immune responses to mental stress in humans. Psychosom Med 1995;57:366-72.

30. Livnat S, Felten SY, Carlson SL, Bellinger DL, Felten DL. Involvement of peripheral and central catecholamine systems in neural-immune interactions. J Neuroimmunol 1985; 10:5-30.

31. Kozian A, Schilling T, Fredén F, et al. One-lung ventilation induces hyperperfusion and alveolare damage in the ventilated lung: an experimental study. Br J Anaesth 2008;100: 549-59.

32. Cheng YJ, Chan KC, Chien CT, Sun WZ, Lin CJ. Oxidative stress during 1-lung ventilation. J Thorac Cardiovasc Surg 2006;132:513-8.

33. De Conno E, Steurer M, Wittlinger M, et al. Anestheticinduced improvement of the inflammatory response to onelung ventilation. Anesthesiology 2009;110:1316-26.

34. Perrini P, Pieri F, Montemurro N, Tiezzi G, Parenti GF. Thoracic extradural haematoma after epidural anaesthesia. Neurol Sci 2010;31:87-8.

35. Tsui BC, Kulkarni P. Migration of a thoracic epidural catheter into the intercostal space via the intervertebral foramen. Anesth Analg 2009;108:371-3.

36. Amagasa S, Igarashi A, Yokoo N, Sato M. Intercostal bleeding that developed during thoracic epidural catheterization. J Anesth 2008;22:197-8.

\section{Member and Individual Subscriber Access to the Online Annals}

The address of the electronic edition of The Annals is http://ats.ctsnetjournals.org. If you are an STS or STSA member or a non-member personal subscriber to the print issue of The Annals, you automatically have a subscription to the online Annals, which entitles you to access the full-text of all articles. To gain full-text access, you will need your CTSNet user name and password.

Society members and non-members alike who do not know their CTSNet user name and password should follow the link "Forgot your user name or password?" that appears below the boxes where you are asked to enter this information when you try to gain full-text access. Your user name and password will be e-mailed to the e-mail address you designate.

In lieu of the above procedure, if you have forgotten your
CTSNet username and/or password, you can always send an email to CTSNet via the feedback button from the left navigation menu on the homepage of the online Annals or go directly to http://ats.ctsnetjournals.org/cgi/feedback.

We hope that you will view the online Annals and take advantage of the many features available to our subscribers as part of the CTSNet Journals Online. These include inter-journal linking from within the reference sections of Annals' articles to over 350 journals available through the HighWire Press collection (HighWire provides the platform for the delivery of the online Annals). There is also crossjournal advanced searching, eTOC Alerts, Subject Alerts, Cite-Track, and much more. A listing of these features can be found at http://ats.ctsnetjournals.org/help/features.dtl.

We encourage you to visit the online Annals at http:// ats.ctsnetjournals.org and explore. 